\title{
Drop Metastasis of Adrenocorticotropic Hormone-Producing Pituitary Carcinoma to the Cauda Equina
}

\author{
Kenichi Takeuchi, Yoko Hagiwara, Koichi Kanaya, Keiji Wada, Masahiro Shiba, Yoshiharu Kato \\ Department of Orthopaedic Surgery, Tokyo Women's Medical University, Tokyo, Japan
}

The diagnosis of pituitary carcinoma cannot be made easily histologically, and most cases of pituitary carcinoma are diagnosed only after the clinical detection of metastasis. Distant metastasis of pituitary tumor occurs in $0.1 \%$ to $0.2 \%$ of cases and has been reported in the liver, bone and central nervous system, with only one case of metastasis to the cauda equine reported. This study describes a rare case of the drop metastasis of adrenocorticotropic hormone-producing pituitary adenocarcinoma to the cauda equina, causing cauda equina syndrome.

Keywords: Drop metastasis; Adrenocorticotropic hormone; Pituitary carcinoma; Cauda equine

\section{Introduction}

Most cases of spinal cord paralysis due to metastatic tumor are caused by epidural metastasis that spreads from vertebral metastasis. In very rare cases, spinal cord paralysis can also result from carcinomatous meningitis caused by intradural metastasis. Diagnosis of pituitary adenocarcinoma cannot easily be made histologically, and some cases of diagnosed benign pituitary adenoma have been shown to develop subsequent distant metastasis. Therefore most cases of pituitary adenocarcinoma are diagnosed only after the clinical detection of metastasis. Distant metastasis of pituitary tumor occurs in $0.1 \%$ to $0.2 \%$ of cases [1] and has been reported mostly in the liver, bone and central nervous system, with only one case of metastasis to the cauda equina reported [2]. This report describes a rare case of drop metastasis of adrenocorticotropic hormone (ACTH)-producing pituitary ad- enocarcinoma to the cauda equina, causing cauda equina syndrome.

\section{Case Report}

Our patient was a 57-year-old male who complained of weakness and pain in the lower extremities, as well as urinary retention. He had undergone transnasal surgery for ACTH-producing pituitary tumor in 1998. He subsequently experienced repeated episodes of residual tumor growth and underwent multiple sessions of tumor resection and radiotherapy. He developed urinary bladder dysfunction and pain/weakness in the lower extremities without a known cause. With gradual worsening of symptoms, the patient presented to our department on referral in 2009. On admission the physical examination revealed that the femoral nerve stretch test was positive only on the right side, and the straight leg-raising test was posi-

Received May 16, 2013; Revised Sep 19, 2013; Accepted Oct 19, 2013

Corresponding author: Koichi Kanaya

Department of Orthopaedic Surgery, Tokyo Women's Medical University,

8-1 Kawada-cho, Shinjuku-ku, Tokyo 162-8666, Japan

Tel: +81-3-3353-8111 (38333), Fax: +81-3-3354-7360, E-mail: Makiichi2@aol.com 
tive at 10 degrees for both legs. In the manual muscle test, reduced muscle strength was observed in the left gastrocnemius muscle and left extensor hallucis longus muscle, and a marked decrease in perception was noted in the L4, L5, and S1 regions on both sides. A urethral catheter was in place due to urinary retention. Neurological findings suggested damage to the cauda equina at L4 and lower levels. ACTH level was high at $356.8 \mathrm{pg} / \mathrm{mL}$ (Fig. 1). On magnetic resonance imaging (MRI), the signal intensity of the lesion was intermediate on T1-weighted images, low to intermediate on T2-weighted images and intermediate with partially high-intensity areas on short-inversion-time inversion recovery images. On a gadolinium-enhanced

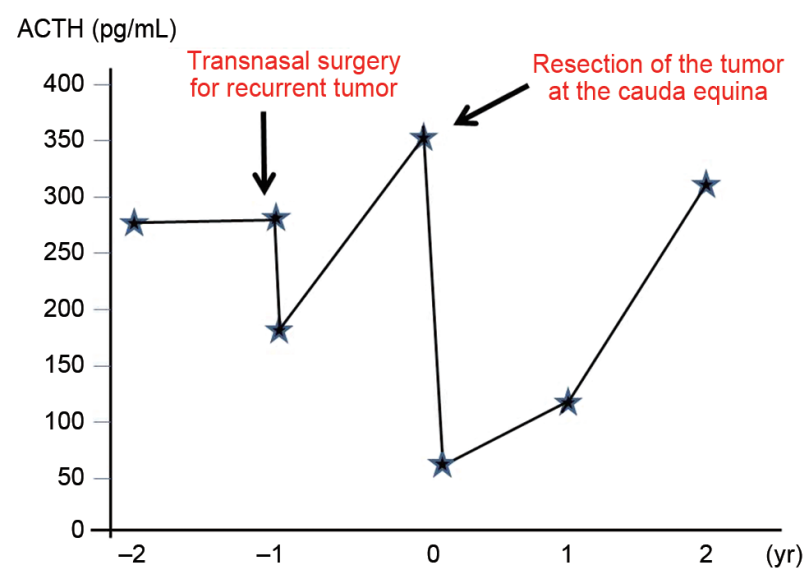

Fig. 1. Changes in adrenocorticotropic hormone (ACTH) level over time. ACTH levels decreased from $356.8 \mathrm{pg} / \mathrm{mL}$ preoperatively to 59.6 $\mathrm{pg} / \mathrm{mL}$ postoperatively. image, a neoplastic lesion was observed, whose content was slightly and irregularly enhanced by the contrast medium (Fig. 2). Given a diagnosis of cauda equina syndrome caused by a tumor in the cauda equina, laminectomy at L3-S2 followed by tumor removal was performed. The tumor was identified as an elastic, hard and clearly bordered mass entangled in several cauda equina nerves and encapsulated by a thin membrane. The tumor was detached from the nerves and as much of it was removed as possible. Two tumor masses were excised (Fig. 3).

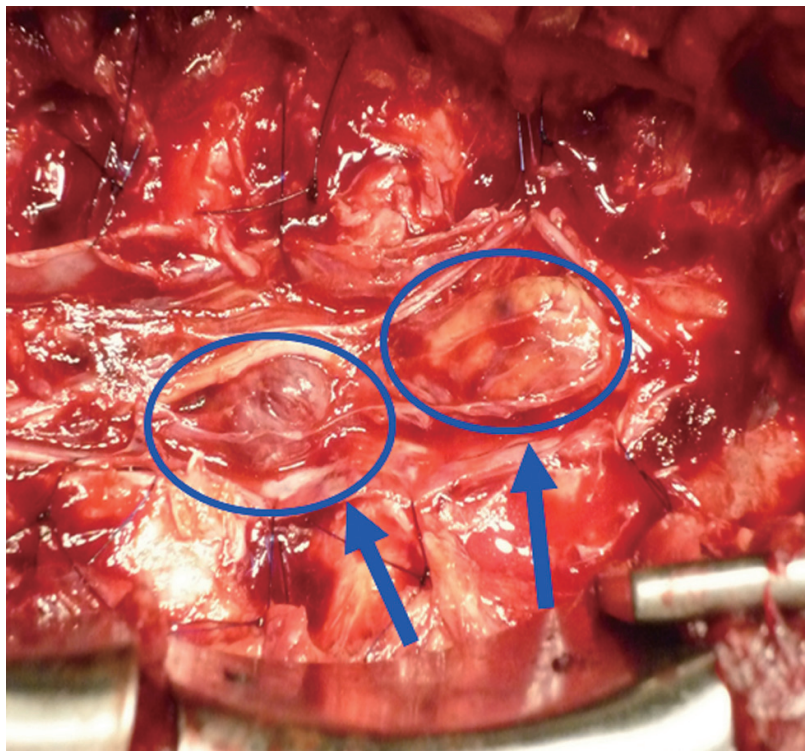

Fig. 3. Surgical findings. The tumor was identified as an elastic, hard and clearly bordered mass encapsulated by a thin membrane and entangled in several cauda equina nerves (arrows indicate tumor).
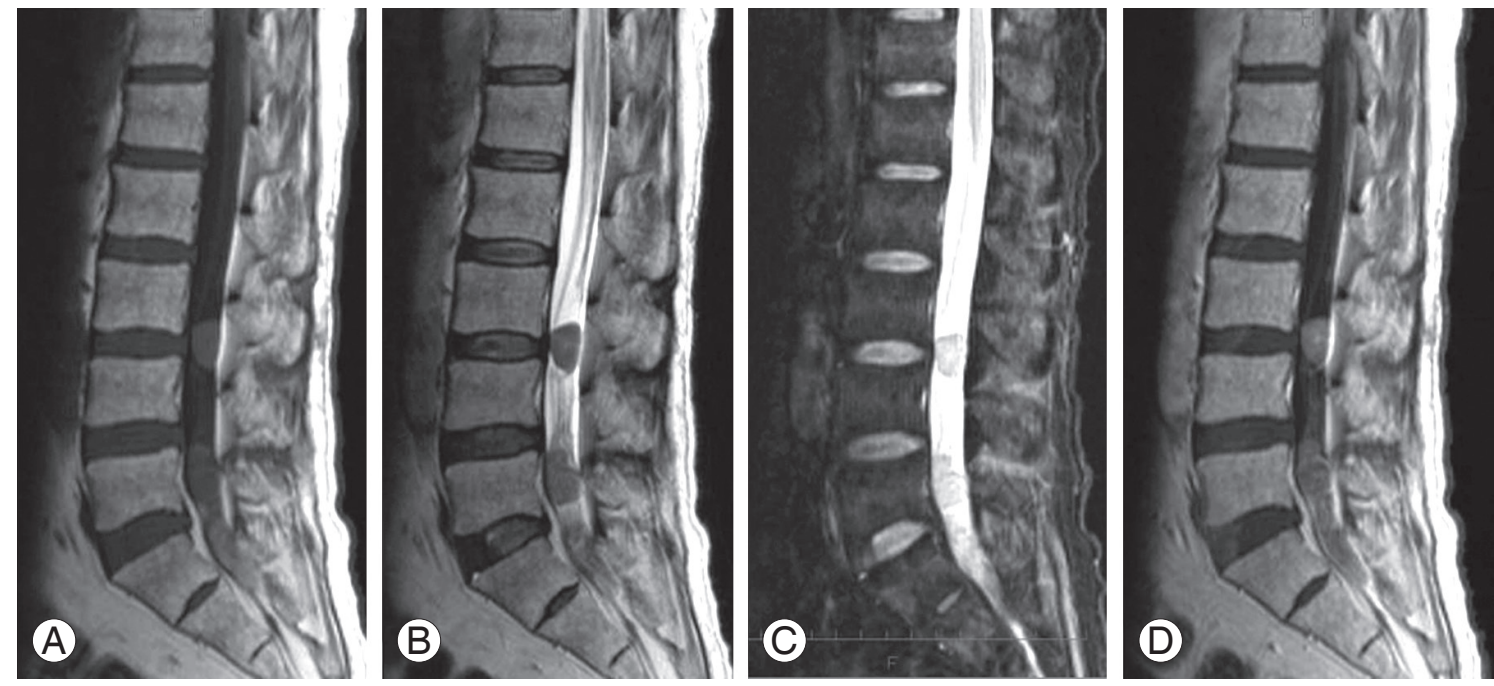

Fig. 2. Preoperative magnetic resonance imaging findings. Signal intensity: intermediate on T1-weighted images (A); low to intermediate on T2-weighted images (B); and intermediate with partially high-intensity areas on short inversion time inversion recovery (C). Neoplastic lesion with gadolinium-enhancement. 
On histopathology, H\&E staining showed proliferation of glandular tissue, with little nuclear atypia and no mitosis. On immunostaining, the mindbomb homolog 1 (MIB-1) index was determined to be less than $3 \%$, and tumor cells were strongly stained for ACTH. As the pathological findings was the same as that from the initial surgery, the tumor was diagnosed as distant metastasis of the pituitary adenocarcinoma (Fig. 4). After tumor removal, the increased ACTH level observed preoperatively was normalized $(59.6 \mathrm{pg} / \mathrm{mL}$ ) (Fig. 1). Two years after surgery, although the patient was still experiencing voiding difficulty, the weakness/pain in the lower extremities had disappeared. The patient has had a favorable postoperative course with no local recurrence (Fig. 5). He is being followed closely due to the possibility of new distant metastases with rising levels of ACTH.

\section{Discussion}

The reported case involved the surgical treatment of drop metastasis of ACTH-producing pituitary carcinoma to the cauda equina. In addition to the fact that the distant metastasis of pituitary tumor is relatively uncommon, the

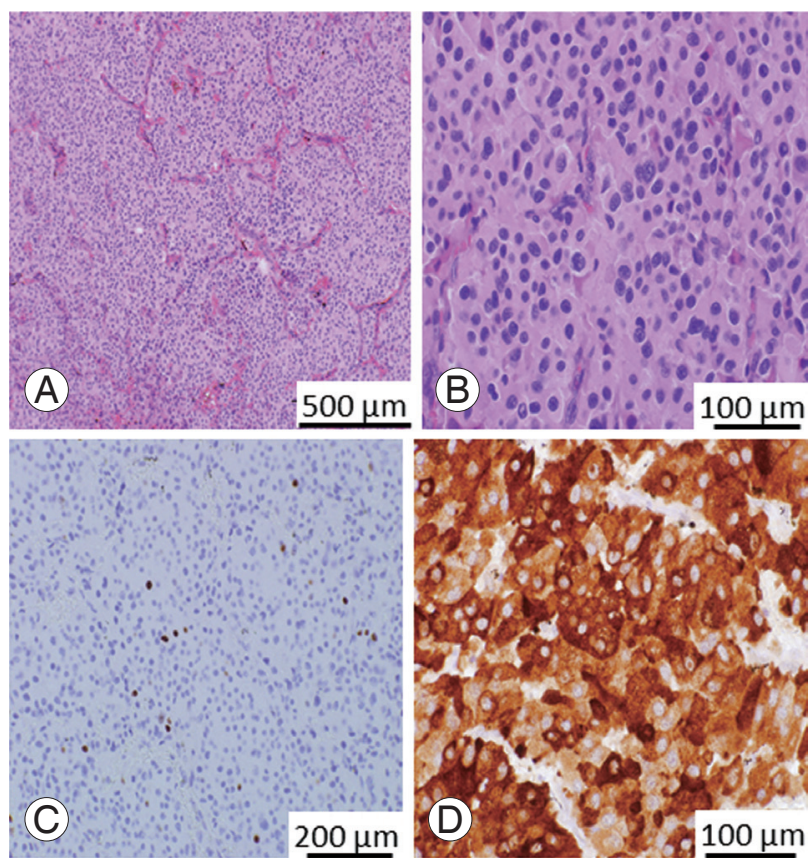

Fig. 4. Histopathological findings. $H \& E$ staining at $\times 10(A)$ and $\times 40(B)$ magnification; immunostaining for mindbomb homolog-1 (MIB-1) (C); and immunostaining for adrenocorticotropic hormone (ACTH) (D). On immunostaining, the MIB-1 index was $<3 \%$, and tumor cell cytoplasm stained strongly for ACTH. present case is rare in terms of the location and mode of metastasis, as it is only the second reported case of pituitary tumor that has metastasized to the cauda equina.

An intradural, extramedullary metastasis occurring in the spinal cord is referred to as meningeal dissemination. It can be categorized into seeding metastasis and drop metastasis, according to extension pattern. Seeding metastasis has been suggested to occur through mechanisms similar to those for central nervous system malignancies, including: 1) hematogenous infiltration through arachnoid blood vessels; 2) direct infiltration from the skull or vertebral metastases; 3 ) transportation from veins with few venous valves, such as those in Batson's plexus; 4) infiltration along nerves; 5) extension through peri-neural or peri-vascular lymph; and 6) drop from metastases in the choroid plexus or near the ventricular system [3-5]. Drop metastasis is considered to occur as a tertiary metastasis of brain metastasis, induced by factors such as gravity in the subarachnoid space and the circulation of cerebrospinal fluid. This pattern of metastasis has been reported for melanoma, lymphoma, small cell lung cancer, breast cancer, colorectal cancer and kidney cancer [3-5].

Characteristic imaging findings of seeding metastasis include multiple enhanced nodular lesions in the spinal canal on contrast-enhanced MRI, disseminated lesions spreading diffusely in the form of membrane and linear

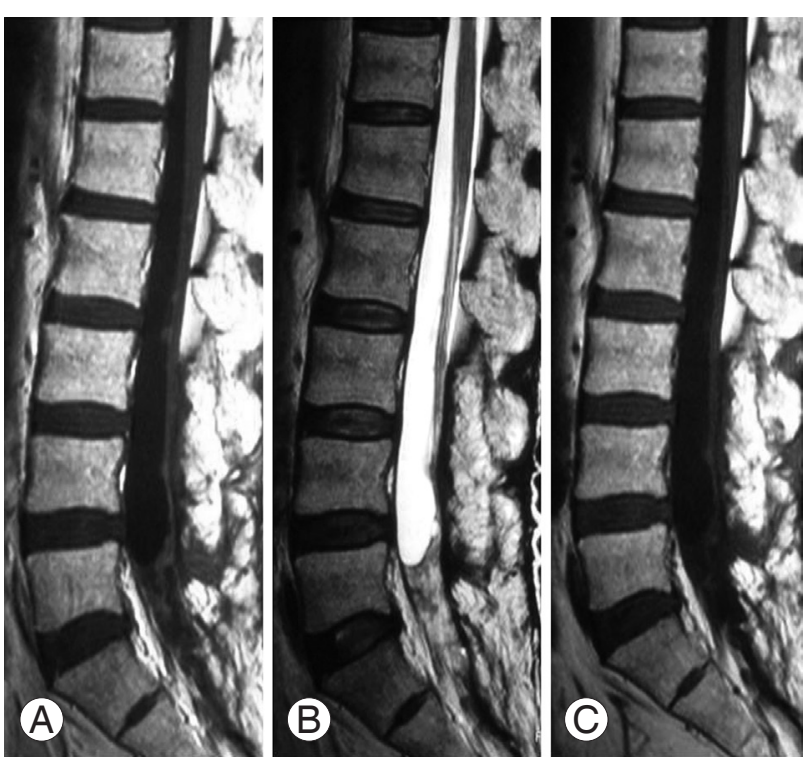

Fig. 5. Magnetic resonance imaging findings two years after surgery. No local recurrence was detected on T1-weighted (A), T2-weighted (B) or gadolinium-enhanced images. 
enhancement of thickened meninges. In contrast, drop metastasis is visualized as a large tumor mass around a spinal nerve root (including the cauda equina). The present case was considered to be drop metastasis, based on imaging and surgical findings.

Malignant tumors undergoing drop metastasis are detached from each other due to the loss of cell-cell adhesion, and they are released into the cerebrospinal fluid without anchorage dependence. For these tumor cells to metastasize, they must acquire an engrafting capacity specific to the spinal cord and nerve roots, such as angiogenic capacity, and drift down to the appropriate sites. In some cases of pituitary adenocarcinoma with distant metastasis, histopathological findings of metastasis include increased MIB-1 index and histological evidence of malignant transformation, compared with pathological findings from the initial surgery [1,6-9]. In the present case, histological examination of the metastasis, as well as that performed after the initial surgery, provided a low MIB-1 index and revealed no nuclear atypia or other evidence of malignancy.

van der Klaauw et al. [1] reviewed 58 cases of pituitary tumor with distant metastasis and reported its incidence to be low at $0.1 \%$ to $0.2 \%$. In these cases, spinal dissemination was observed in one case. Spinal metastasis was identified in the spine in four cases, the spinal cord in three cases, the spinal subarachnoid space in three cases, the spinal meninges in two cases and the cauda equine in one case [2]. Thus case reports of drop metastasis to the cauda equina are very rare. Given the increased frequency of recurrence at the metastatic site and shortened time to re-growth after radiotherapy in the present case, it is possible that the tumor in the present case had an increased potential for metastasis which has not been characterized. The 1-year survival rate of patients with metastatic ACTH-producing pituitary carcinoma is $33 \%$. For this type of tumor, surgical treatment should be selected as the first choice whenever possible [1]. The present case was diagnosed as drop metastasis, and the patient has survived for 3 years after surgery thus far, although the tumor was resected only as much as possible. However, since tumor cells are disseminated in the dural space, the patient is still at a high risk of tumor regrowth and is be- ing followed carefully.

In summary, we have reported a rare case of drop metastasis of ACTH-producing pituitary carcinoma to the cauda equina.

\section{Conflict of Interest}

No potential conflict of interest relevant to this article was reported.

\section{References}

1. van der Klaauw AA, Kienitz T, Strasburger CJ, Smit JW, Romijn JA. Malignant pituitary corticotroph adenomas: report of two cases and a comprehensive review of the literature. Pituitary 2009;12:57-69.

2. Pernicone PJ, Scheithauer BW, Sebo TJ, et al. Pituitary carcinoma: a clinicopathologic study of 15 cases. Cancer 1997;79:804-12.

3. Chow TS, McCutcheon IE. The surgical treatment of metastatic spinal tumors within the intradural extramedullary compartment. J Neurosurg 1996;85:22530.

4. Jacobs WB, Perrin RG. Evaluation and treatment of spinal metastases: an overview. Neurosurg Focus 2001;11:e10.

5. Schick U, Marquardt G, Lorenz R. Intradural and extradural spinal metastases. Neurosurg Rev 2001;24:15.

6. Kouhara $\mathrm{H}$, Tatekawa $\mathrm{T}$, Koga $\mathrm{M}$, et al. Intracranial and intraspinal dissemination of an ACTH-secreting pituitary tumor. Endocrinol Jpn 1992;39:177-84.

7. Lubke D, Saeger W. Carcinomas of the pituitary: definition and review of the literature. Gen Diagn Pathol 1995;141:81-92.

8. Thapar K, Kovacs K, Scheithauer BW, et al. Proliferative activity and invasiveness among pituitary adenomas and carcinomas: an analysis using the MIB-1 antibody. Neurosurgery 1996;38:99-106.

9. Taya K, Terao T, Nakazaki H, et al. Intracranial metastasis of pituitary adenoma: a case report. No Shinkei Geka 2004;32:279-84. 